\title{
VALEURS SÉMIOLOGIQUES DES MARQUEURS TESTICULAIRES
}

\author{
C. B. Barthelemy* \\ *Unité de reproduction CHU BRETONNEAU - 37044 TOURS Cedex
}

SEMIOLOGIC VALUES OF THE TESTICULAR MARKERS. Several proteins have been determined in human seminal plasma in order to study male infertilities more accurately. Some of them, as androgen binding protein, somatomedine $\mathrm{C}$, epidermal growth factor, plasminogen activator and ceruloplasmine are not reliable markers against testbumine, transferrine and LDHX. We particularly comment about transferrine, which, as LDHX, presents a strong correlation with sperm count. Nevertheless, differential diagnosis of azoospermia can't be done by means of one of these markers. Key words : testicular markers, infertility, testbumine, transferrine, LDHX, ABP, EGF, Plasminogen Activator, Ceruloplasmine. Andrologie, 1991, $1: 11-12$

Depuis plusieurs années déjà, de nombreuses tentatives, la plupart du temps infructueuses ont été effectuées, afin d'essayer de trouver "in vivo" des indices spécifiques de la fonction testiculaire dans le plasma séminal humain afin d'essayer d'éciaircir le diagnostic des infertilités masculines.

Cette difficulté est due principalement à la complexité de la spermatogénèse, processus qui implique l'interaction de nombreuses cellules, un contrôle hormonal complexe, de nombreux facteurs de croissance, intervenant sur une période relativement longue (1).

Le plasma séminal est un mélange de secrétions de diverses origines : testiculaires, épididymaires, prostatiques, vésiculaires et sériques. L'éjaculat est donc le résultat d'une série de sécrétions, migrations, réabsorptions, maturations. Sa composition varie selon les individus, avec le moment, l'âge et différents facteurs intercurrents. Nous y trouvons de 100 à 200 protéines ou peptides dont le dosage va impliquer obligatoirement la nécessité de connaître leur origine pour évaluer la contribution relative de chaque compartiment.

Dans les tubes séminifêres coexistent 2 grands types cellulaires :

les cellules germinales dont le rendement peut être quantifié par la biopsie testiculaire et surtout

Communication présentée au ${ }^{\text {cme }}$ congrès de la SALF, Sousse, Sept. 1990 par la production de spermatozoïdes, lors de leur apparition dans l'éjaculat après leur passage dans l'épididyme. Ces dernières produisent aussi la LDH X ou C4 marqueur que l'on peut mettre en évidence dans le plasma séminal.

Et surtout les cellules de Sertoli : cellules somatiques de l'épithélium séminifere, cellules clé du contrôle de la spermatogénèse puisqu'elles fournissent à la fois un support physique et métabolique aux cellules germinales en voie de développement par contact direct et par le biais de leur activité sécrétoire. En effet les complexes jonctionnels des cellules de Sertoli créent une barrière hémotesticulaire qui empêche l'entrée des protéines sériques et d'autres macromolécules dans le compartiment tubulaire (et vice versa). La composition du fluide (lactate, pyruvate, ions, stéroïdes, nombreuses protéines, enzymes ou facteurs de croissance...) est donc limitée à la contribution sécrétoire des cellules de Sertoli. Des études sophistiquées in vitro ont permis l'identification de nombreuses protéines dont certaines sont spécifiques du testicule et d'autres identiques aux protéines sériques. Certaines peuvent être retrouvées dans le plasma séminal et y ont été dosées.

\section{ABP : Androgen binding protein (10)}

L'ABP est la première protéine d'origine testiculaire qui a été isolée. Elle est secrétée à la fois au pôle basal ( 20 à $33 \%$ ) et au pôle apical de la cellule. Elle transporte les androgènes (testostérone et DHT) aux cellules germinales et dans le fluide testiculaire. La quantité dans le plasma séminal est faible puisqu'il existe une réabsorption très importante (95\%) au niveau du segment initial de la tête de l'épididyme. Elle existe sous 2 formes, I et II. La forme II est pratiquement identique à la $T e B G$, donc difficilement dissociable de celle-ci au dosage. C'est la raison pour laquelle nous n'avons pas retrouvé de tentatives de dosage postérieures à celle du Plymate, car il semble difficile d'évaluer ce que l'on dose effectivement.

\section{EGF : epidermal growth factor (4)}

Facteur de croissance synthétisé par la Sertoli. Les études effectuées dans le plasma séminal montrent une concentration supérieure à celle du sang. Elles comparent des populations dhommes fertiles et infertiles. Les taux varient de $41 \mathrm{ng} / \mathrm{ml}$ à $53 \mathrm{ng}$ chez les infertiles et de $41 \mathrm{ng}$ à $48 \mathrm{ng}$ chez les hommes fertiles. Il n'y a aucune corrélation avec la numération des gamètes : ce n'est donc pas un bon marqueur de la fonction testiculaire.

Activateurs du plasminogène (6)

Ce sont des protéases aussi secrétées par la Sertoli, présentant une activité fibronolytique : elles transforment le zymogène et le plasminigène en plasmine. Elles seraient indispensables à la translocation des spermatocytes du compartiment basal au compartiment adluminal, ainsi qu'au relargage des spermatozoïdes matures lors de la spermiation.

Dans le plasma séminal 2 types sont représentés : type urokinase uPA et type tissulaire tPA. Ce dernier est le plus important du point de vue physiologique : $(480 \mathrm{ng} / \mathrm{ml}$ au lieu de $7 \mathrm{ng})$. L'étude faite par Mac Gregor (6) portait sur 42 hommes infertiles. Il n'a pas retrouvé de corrélation avec les différents paramètres du sperme étudiés et la fertilité ultérieure des sujets. Ceci confirme l'origine probablement prostatique de ceux-ci.

\section{Ceruloplasmine (7)}

Cette protéine secrétée par la Sertoli est identique à la protéine sérique. Elle transporte le $\mathrm{Cu}^{++}$jusqu'aux cellules germinales. Elle est en quantité 25 fois inférieure à celle de la transferrine ( 1 à $6 \mu \mathrm{g} /$ éjaculat). Orlando a montré dans son étude que la céruloplasmine présente dans le plasma séminal venait principalement de la circulation puisque les taux étaient identiques chez les hommes fertiles $(4,21)$ et vasectomisés $(4,27)$. Il en conclut que ce dosage n'a aucune valeur dans le plasma séminal pour le diagnostic de linfertilité.

\section{Somatomedin C ou IGF 1 :} Insulin growth factor (2)

Peptide secrété par la Seroli et peut-être l'épididyme. C'est un facteur de croissance qui stimule la synthèse de DNA des cellules de nombreuses espèces animales. Le taux est faible dans le plasma séminal : $20 \mathrm{ng} / \mathrm{ml}$ chez les hommes fertiles, significativement diminué chez les azoospermes $9 \mathrm{ng} / \mathrm{ml}(\mathrm{P}<0,02)$. Mais dans ces études il existe de nombreux recouvrements des valeurs entre hommes fertiles et vasectomisés. De plus il n'y a pas de corrélation avec la Transferrine et la FSH : ce n'est donc pas un index fiable de la fonction testiculaire.

Tesbumine : albumine like protéine (9)

Elle est immunologiquement et structuralement 
identique à la sérum-albumine. Elle est synthétisée par les cellules de Sertoli uniquement dans les tubes séminiferes adultes. Elle sert probablement au transport des androgènes (testostérone). Orlando a montré que, comme la Transferrine, 80 $\%$ de l'albumine séminale était d'origine testiculaire. Il existe une bonne corrélation avec la numération $(r=0,42)$ et la Transferrine, mais la diminution des taux est moins sensible pour les oligozoospermies ( $71 \%$ ) et les azoospermies (59\%) : inconvénient important selon l'auteur pour être un marqueur très sensible.

\section{Transferrine}

C'est un marqueur que notre équipe a étudié plus particulièrement. C'est une $B$ glycoprotéine de PM 79000 synthétisée avec l'aide de la FSḦH par la cellule de Sertoli. Elle représente environ 5\% des protéines nouvellement fabriquées par la Sertoli. Elle se différencie de la Transferrine sérique par ses glycosylations. Elle transporte les ions $\mathrm{Fe}^{\mathrm{et+}} \mathrm{à}$ l'épithélium germinal en développement car des récepteurs ont été localisés sur les spermatocytes pachytènes et les jeunes spermatides (5). Des études ont rapporté une quantité importante de Transferrine dans le plasma séminal humain : 80\% de celle-ci serait d'origine sertolienne (5).

Nous avons étudié ce marqueur potentiel à l'aide d'un dosage RIA mis au point au laboratoire d'immunologie du CHU de Tours (Pr Bardos), sur une population d'hommes infertiles (azoospermies, oligozoospermies, normospermies), de donneurs fertiles et une population d'azoospermies présentant plus de 10 leucocytes par ml.

Notre étude confirme que $80 \%$ de la transferrine du plasma séminal est d'origine sertolienne. Sa concentration est très significativement élevée chez les normospermes et les oligospermes par rapport aux azoospermes et aux oligospermies très sévères $(<1$ million). Il existe une corrélation très forte $(r=0,72)$ avec la numération des gamètes ainsi qu'une corrélation négative avec la $\mathrm{FSH}$ plasmatique $(\mathrm{r}=0,29$ $\mathrm{p}<0,01)$ : plus la FSH est élevée, plus la Transferrine est basse. Aucune corrélation n'a été retrouvée avec d'autres paramètres spermatiques: tératozoospermie ou cellules de la lignée germinale. Mais surtout les valeurs de la Transferrine ne sont pas différentes dans les cas d'azoospermies sécrétoires ou excrétoires.

Les résultats sont identiques à ceux d'autres études (5) pour la corrélation avec la numération, mais contrairement à eux nous avons trouvé à deux reprises une corrélation avec la FSH plasmatique. A notre grand regret, à la différence de Dadoune, nous n'avons pu établir de diagnostic différentiel des azoospermies avec la Transferrine séminale. Toutefois il semble que les taux de Transferrine soient nettement pathologiques dans les processus infectieux ou inflammatoires.

La Transferrine semble un index plus révélateur de l'aspect quantitatif plutôt que qualitatif de la spermatogénèse, c'est un indicateur assez fiable de la dysfonction testiculaire, mais elle ne précise pas l'origine du désordre.

\section{LDH X (8)}

Cette enzyme, produite par les cellules germinales, est une isoenzyme de la $\mathrm{LDH}$ spécifique du testicule humain post-pubertaire. Elle est le seul marqueur originaire à $100 \%$ du testicule. Elle facilite l'utilisation du lactate produit par les cellules de Sertoli. Elle catalyse la conversion lactate-pyruvate par l'intermédiaire de la régulation de FSH et insuline. Cette enzyme est donc vitale pour les processus métaboliques générateurs d'énergie pour la survie du gamète.

3 études ont rapporté les résultats de leurs dosages (Garella et al 1982, Eliasson et al 1985, Orlando et al 1988, in 8). Il existe une forte corrélation avec la numération ( $1 / 3$ du taux des normaux chez les oligospermes) mais pas avec la FSH, la Transferrine, ou tout autre paramètre spermatique. De plus la LDH X est indétectable chez les azoospermes et dans les oligospermies très sévères. Elle apparaît plutôt comme un marqueur post-méiotique de l'activité testiculaire.

En conclusion, la Transferrine et la LDH X semblent être les moins mauvais marqueurs "in vivo" de la fonction testiculaire parmi tous ceux étudiés. Elles peuvent permettre un suivi de certains essais pharmacologiques (gonadotrophines, $\mathrm{GnRH}$ ), ou antiinflammatoires pour la Transferrine. A l'heure actuelle nous ne possédons pas de marqueur testiculaire précis pour le diagnostic de l'azoospermie.

Si l'on connaît bien les interactions ultrastructurales entre toutes les cellules du tube séminifêre, il y a encore beaucoup à apprendre sur le contrôle physiologique et biochimique de la spermatogénèse tant ces interactions cellulaires sont complexes et variables. Cest donc une tâche ardue que d'estimer les causes testiculaires de l'infertilité masculine. L'avenir est peut-être dans la séparation spécifique de ces protéines dans le plasma séminal et/ou l'utilisation d'anticorps monoclonaux, ou dans la biologie moléculaire et ses sondes à DNA.

\section{REFERENCES}

1 - Bardin C W, Cheng C Y, Musto N A, Gunsalus G L: The Sertoli cells. In Knobil, E. Neill J. (Eds). The physiology of Reproduction. Raven Press Ltd, New
Yok, 1988, 933-974

2 - Barnit. C., Orlando C., Caldini A.L., Casano R., Natali A., Chelo E. J., Bonfanti L., Forti T., Serio M. Immunoassayable somatomedin $\mathrm{C}$ in seminal plasma of azoospermic men. Int. J. Androl., 1987, $10: 457-462$

3 - Barthelemy C., Khalfoun B., Guillaumin J.M., Lecomte P., Bardos P. Seminal fluid transferrin as an index of gonadal function in man. J. Reprod. Fertil. 1988, 82:113-118

4 - D'Cruz O.J., Haas G.G. : Immunoreactive human epidermal growth factor in human seminal plasma. J. Clin. Endocrinol. Metab., 1989, 68 : 1136-1140

5 - Holmes S.D., Lipshultz L.I., Smith R.B. Transferrin and gonadal dysfunction in man. Fertil. Steril., 1982 $38: 600-604$

6 - Mac Gregor I.R., Hargreave T.B., Elton R.A. Seminal plasma plasminogen activator and fertlity. Andrologia, 1987, 4 : 452-455

7 - Orlando C., Caldini A.L., Barni T., Wood W.G., Strasburger C.J., Natali A., Mayer A., Forti G., and Serio M. Ceruloplasmin and transferrin in human seminal plasma are they an index in seminiferous tubular function? Fertil. Steril., 1986, $43: 290-294$

8 - Orlando C., Casano R., Caldini A.L., Forti T., Barni T., Bonfanti L., Serio M. Measurement of seminal LDH X and transferrin in normal and infertile men. J. Androl. 1988, $9: 220-223$

9 - Orlando C., Casano R., Forti T., Barni T., Vanelli G.B., Baldoni G.C., Serio M. Immunologically reactive albumine like protein in human testis and seminal plasma. J. Reprod. Fertil., 1988, $13: 687$ 692

10 - Plymate S.R., Fariss B.L., Smith M.L., Jacob W.H., Matej L.A. : Seminal fluid androgen binding protein. Andrologia, 1981, 13:308-313

RESUME : De nombreuses protéines ont été dosées dans le plasma séminal humain pour étudier plus précisément les infertilités masculines. Certaines, comme l'androgen binding protein, la somatedine $\mathrm{C}$, l'epidermal growth factor, l'activateur du plasminogène et la ceruloplasmine ne sont pas des marqueurs fiables au contraire de la testbumine, de la transferrine et de la LDHX. Nous évoquons plus particulièrement la transferrine, qui comme la LDH X présente une forte corrélation avec la numération des spermatozoïdes. Par contre le diagnostic différentiel des azoospermies ne peut être fait avec aucun de ces marqueurs. Mots clés : marqueurs testiculaires, infertilité, tesbumine, transferrine, LDHX, ABP, EGF, activateur de plasminogène, ceruloplasmine.

Andrologie, 1991, 1 :11-12 